\title{
Oxygen nanobubbles enhanced photodegradation of oxytetracycline under visible light: synergistic effect and mechanism
}

Lei Wang ${ }^{\dagger}$, a, Jafar Ali ${ }^{\text {a, b }}$, Zhibin Wang ${ }^{\dagger}, \mathrm{c}$, N. A. Oladoja ${ }^{\mathrm{d}}$, Rong Cheng *, e , Changbo

Zhang *, f, Gilles Mailhot ${ }^{\mathrm{g}}$, Gang Pan *, a, h

${ }^{\text {a }}$ Key Laboratory of Environmental Nanotechnology and Health Effects, Research Center for Eco-environmental Sciences, Chinese Academy of Sciences, 18 Shuangqing Road, Beijing 100085, P. R. China

${ }^{\mathrm{b}}$ University of Chinese Academy of Sciences, Beijing 100049, P. R. China

${ }^{c}$ Beijing Advanced Sciences and Innovation Center, CAS, No.5, 2nd East Yanqi, Haircut District, Beijing, 101407, China

${ }^{\mathrm{d}}$ Hydrochemistry Research Laboratory, Department of Chemical Sciences, Adekunle Ajasin University, Akungba Akoko, Nigeria

${ }^{\text {e }}$ School of Environment and Natural Resources, Renmin University of China, Beijing 100872, China

${ }^{\mathrm{f}}$ Agro-environmental Protection Institute, Ministry of Agriculture and Rural Affairs, Tianjin 300191, P. R. China

g CNRS, SIGMA Clermont, Institut de Chimie de Clermont-Ferrand, Université Clermont Auvergne, F-63000 Clermont-Ferrand, France

${ }^{\mathrm{h}}$ Centre of Integrated Water-Energy-Food Studies, School of Animal, Rural and Environmental Sciences, Nottingham Trent University, Brackenhurst Campus, Southwell NG25 0QF, United Kingdom

${ }^{\dagger}$ Co-first author with the same contribution to this work. 
* Corresponding author: chengrong@ruc.edu.cn (R. Cheng); zhchb1976@163.com (C.B. Zhang); gpan@rcees.ac.cn (G. Pan)

\section{Abstract}

Nanobubbles have gained much attention due to their unusual properties such as large specific surface area, surface energy and internal energy, thereby, increase the surface reaction and improve the mass transfer efficiency. The long term stability of nanobubbles and generation of reactive oxygen species enables them to address various environmental issues for water purification without secondary pollution. However, there are few studies concerning the application of oxygen nanobubbles in the advanced oxidation processes (AOPs). In this study, first time, oxygen nanobubbles were introduced into the photoreaction system under visible light irradiation for enhanced photodegradation of oxytetracycline (OTC). Results indicated that oxygen nanobubbles significantly improved the photodegradation of OTC through high mass transfer and generation of reactive radicals during the nanobubble collapse. The oxygen nanobubble stability was confirmed to be $\mathrm{pH}$ dependent. The nanobubbles concentration increased from 0.76 to $3.78 \times 10^{8}$ particles $/ \mathrm{mL}$, while the mean size decreased from $205 \mathrm{~nm}$ to 138 $\mathrm{nm}$ as the $\mathrm{pH}$ value increased (3.2-11.0). The degradation efficiency of OTC was improved from $45 \%$ to $98 \%$ with the increase of $\mathrm{pH}(4.0-11.0)$. Oxygen nanobubbles hold promise to improve the AOPs, such as reducing catalysts and chemicals usage and costs. Quenching experiments demonstrated that $\bullet \mathrm{OH}$ was the predominant active species induced the photodegradation of OTC. With the development of nanobubbles 
technology, it is anticipated to realize totally chemicals free green technology. This study provides a novel nanobubble/AOPs technology, which will be more efficient and environmental friendly and will have broad application in waste water treatment and drinking water purification.

Keywords: Oxygen nanobubble; AOPs; Photodegradation; Free radicals; PPCPs; Oxytetracycline

\section{Introduction}

Nanobubbles are defined as gas cavities in the aqueous solution with diameters less than $1 \mu \mathrm{m}$. Interfacial / surface nanobubbles and free bulk nanobubbles are the two prevailing types of ultrafine/nanobubbles [1-3]. Atomic force microscope (AFM) micrographic evidence on the existence of nanobubbles established a significant milestone for nanobubble technology in physicochemical grounds $[4,5]$. Since then, nanobubbles have attracted tremendous attention, which culminated in significant number of research outputs on nanobubbles generation methodologies [6-9], nanobubble nucleation [10], nanobubble dynamic stabilities as a function of different parameters $[11,12]$, nanobubbles properties [13-15] and mechanism of elucidating these properties [16, 17], characterization of nanobubbles [18-21] and potential applications in many fields, which include medical [22-24], industrial [25, 26], agricultural [27-29] and environmental applications [30, 31].

Nanobubbles possess large specific surface area, high surface energy and internal energy, which enhance the surface reaction and improve the efficiency for mass transfer 
$[32,33]$. The life span of nano-sized bubbles (hours to days) is longer than that of macro or microbubbles in the aqueous system [29]. It has been reported that the collapse of micro and nanobubbles generate reactive oxygen species (ROS), which include hydroxyl radicals, singlet oxygen, superoxide [34-37]. These properties were the impetus for the consideration of micro and nanobubbles as a useful technology for water treatment and disinfection $[32,38]$. The cases in which micro and nanobubbles have been applied for pollution management include the remediation of dyes, phenol and alachlor polluted aqua system [33,39]. Moreover, ozone nanobubbles have also showed the more effectiveness than air-nanobubbles in the decomposition of pollutants [40-42]. So far, there is paucity of research reports on the application of nanobubbles in the advanced oxidation processes (AOPs) for pollution remediation [43, 44]. Thus, synergistic effect of oxygen nanobubbles with AOPs and their detailed mechanisms is yet to be elucidated.

Photocatalytic degradation technology is one of the most efficient AOPs in the removal of organic pollutants from wastewater. It has been widely used in water treatment since the development of powdered photocatalyst. However, the use of powdered catalysts are hampered by low reusability in suspension system and less absorption in the visible light region. These factors have hindered the large-scale application and efficiency. In order to overcome these challenges, a more efficient method, to reduce the catalyst dosage or other chemical based oxidant is urgently required. Premised on the peculiar features of nanobubbles, which have been observed to influence the physicochemical properties of the medium in which they are dispersed 
[45], it is considered a veritable tool that can be deployed to improve the current AOPs or enable new technologies for producing less secondary pollutants and achieving more efficient and ecofriendly treatment goals.

Tetracycline (i.e. tetracycline, chlortetracycline and oxytetracycline) is a class of antibiotics with broad-spectrum antimicrobial action against infectious bacteria. They are commonly used in animal feed as growth promoters and prophylactics for swine and cattle production [46]. Excessive usage and low biodegradability of tetracycline can potentially cause serious risk to human and environment. Traditional physical/chemical methods are ineffective in the management and complete removal of tetracycline. Photocatalytic degradation is considered an efficient and attractive method. Although, number of studies have focused on the development of high efficient photocatalysts, include $\mathrm{TiO}_{2} @ \mathrm{GO}$ [47, 48], Au-CuS-TiO 2 [49], $\mathrm{BiVO}_{4}$ [50], MWCNT/BiVO 4 [51], $\mathrm{Ag} / \mathrm{p}-\mathrm{Ag}_{2} \mathrm{~S} / \mathrm{n}-\mathrm{BiVO}_{4} \quad[52], \quad \mathrm{ZnO} / \mathrm{ZrO}_{2} \quad$ [53], $\mathrm{Ag}_{3} \mathrm{PO}_{4} / \mathrm{TiO}_{2} @ \mathrm{MoS}_{2}[54], \mathrm{Ag} / \mathrm{Ag}_{3} \mathrm{VO}_{4} / \mathrm{g}-\mathrm{C}_{3} \mathrm{~N}_{4}$ [55], but nano-catalysts preparation requires high chemical requirements and costs. The problem of recycling is also an issue, which has restricted their practical applications. Therefore, it is necessary to find more efficient and ecofriendly treatment processes to eliminate tetracycline and other antibiotics from the waste stream.

The purpose of this work is to develop a novel catalysts free nanobubble/AOPs technology and investigate the synergistic effect of oxygen nanobubbles on the photodegradation of oxytetracycline (OTC) under visible light. The oxygen nanobubbles in the reaction solution were characterized and the effects of $\mathrm{pH}$, 
nanobubbles size and concentration on the photodegradation process were also evaluated.

\section{Materials and methods}

\subsection{Materials}

Oxytetracycline (OTC) was purchased from Sigma-Aldrich and HPLC grade of methanol and formic acid were obtained from Fisher Chemicals (Beijing, China). 1, 4benzoquinone (BQ), 2-propanol and $\mathrm{NaN}_{3}$ were obtained from Aladdin (Shanghai, China). $\mathrm{NaOH}$ and $\mathrm{HCl}$ were obtained from Sinopharm Chemical Reagent Co., Ltd (China). Pure water was obtained from a Milli-Q system (18.2 M $\Omega$, Millipore Corp., Boston, MA). All reagents were used without further treatment. High-purity oxygen (99.99\%) was used as the gas source for preparing oxygen nanobubbles. Stock solution of (OTC, $10 \mathrm{mM})$ was prepared by dissolving the reagent in the ultrapure water and subsequently stored in refrigerator $\left(4^{\circ} \mathrm{C}\right)$ in the dark.

\subsection{Nanobubbles preparation and characterization}

Oxygen nanobubbles were prepared by the nanobubbles generator (Zhongnong Tianlu micro-nano bubble water technology Co. Ltd, China). Different sizes of nanobubbles were obtained by filtrating with various pore size of teflon membrane $(0.45 \mu \mathrm{m}$ and $0.22 \mu \mathrm{m})$. The bulk nanobubbles concentration and mean size were detected by the nanosight (Nanoparticle tracking analysis, NTA NS500, Malvern Instrument Ltd. UK). The zeta potential of nanobubbles were detected by Zetasizer Nano ZS (Malvern Instrument Ltd. UK). A fiber optic oxygen meter (PreSens, Fibox 4, Germany) was used to measure the variation of dissolved oxygen (DO) during the 
photolysis reaction.

\subsection{Photolysis experiments}

The photolysis experiment was carried out in a $500 \mathrm{~mL}$ open cylindrical reactor, with a 250W tungsten halogen lamp (Philips, JLZ250KN, U4K-PSH, Shanghai Yaming, Co., Ltd), with a wavelength range of $400 \mathrm{~nm}$ to $780 \mathrm{~nm}$. The reaction was conducted at a constant temperature of $25^{\circ} \mathrm{C}$ using a circulating thermostatically controlled water through the outer pyrex glass water-jacket of the reaction vessel (Fig. 1).

Reaction solutions were freshly prepared by dissolving OTC in various reaction system. The reaction system with oxygen nanobubbles was prepared by using nanobubble water, which was freshly generated in ultrapure water by nanobubbles generator and the ultrapure $\mathrm{O}_{2}$ was used as gas sources. The reaction solution with ordinary oxygen aeration was prepared by ultrapure water bubbling with ultrapure $\mathrm{O}_{2}$ about $5 \mathrm{~min}$. Various concentrations of nanobubbles were obtained by diluted the higher concentration of nanobubbles water using the ultrapure water (DO 30mg/L). Thus, these solutions have the similar DO concentrations $(30 \mathrm{mg} / \mathrm{L})$. The initial $\mathrm{pH}$ of reaction solution was adjusted with $1 \mathrm{mM} \mathrm{HCl}$ and $1 \mathrm{mM} \mathrm{NaOH}$. All photoreaction experiments were repeated three times and carried out at the room temperature. 


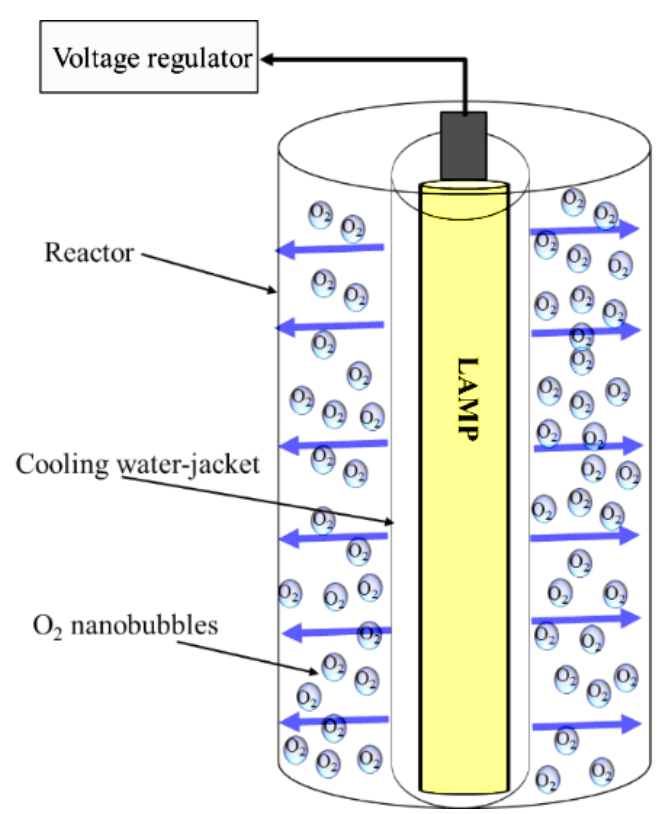

Fig. 1 Scheme of irradiation setup

\subsection{HPLC analysis}

At a given time interval, $3 \mathrm{~mL}$ solution was sampled and filtered through a 0.45 $\mu \mathrm{m}$ cellulose acetate membrane for OTC determination. The concentrations of OTC was analyzed with an Agilent 1200 HPLC system with 2996 Diode Array Detector (Agilent, USA) and XTerra MS C18 column $(3.5 \mu \mathrm{m}, 150 \mathrm{~mm} \times 4.6 \mathrm{~mm})$. The optimized mobile phase was $50 \%$ methanol and $50 \%$ formic acid $(0.02 \%)$ aqueous solution. The photodiode array detector was set at $355 \mathrm{~nm}$. The temperature was set at $25{ }^{\circ} \mathrm{C}$ and the flow rate was $0.5 \mathrm{~mL} / \mathrm{min}$. The photodegradation efficiency was expressed as $\mathrm{C}_{t} / \mathrm{C}_{0}$, where $\mathrm{C}_{0}$ is the initial concentration, and $\mathrm{C}_{\mathrm{t}}$ is the concentration at reaction time $\mathrm{t}$.

Radical quenching experiment was conducted to identify the active species in the photocatalytic reaction. 2-propanol $(1 \mathrm{mM}), \mathrm{NaN}_{3}(1 \mathrm{mM})$ and benzoquinone $(1 \mathrm{mM})$ were used as scavengers for trapping hydroxyl radicals $(\cdot \mathrm{OH})$, singlet oxygen $\left({ }^{1} \mathrm{O}_{2}\right)$ and superoxide radical $\left(\mathrm{O}_{2}{ }^{\circ}\right)$, respectively. All the experiments were conducted in triplicate. 


\section{Results and discussion}

\subsection{Nanobubbles preparation and characterization}

After 10 min generation period, nanobubbles were detected in the solution by the nanoparticle-tracking analysis instrument. The time dependent variations of concentration and size distribution of nanobubbles are presented in Fig. 2. At 240 min, the size distribution of the nanobubble was still in the range of 100-500 $\mathrm{nm}$ and the concentration was around of $2.29-2.51 \times 10^{8}$ particles $/ \mathrm{mL}$. The mean size was $185-210$ $\mathrm{nm}$ and these concentration and size range were maintained, even after 24 hours (Fig. 2 e-e1). Thus, oxygen nanobubbles stability in pure water was confirmed, which was in consistence with previous reports that nanobubbles have long lifetime from hours to months $[29,56-59]$. The long term stability of nanobubbles and other properties such as large specific surface area, surface energy and internal energy, thereby, increase the surface reaction and improve the mass transfer efficiency, which enables them to address various issues for water purification and other applications. 

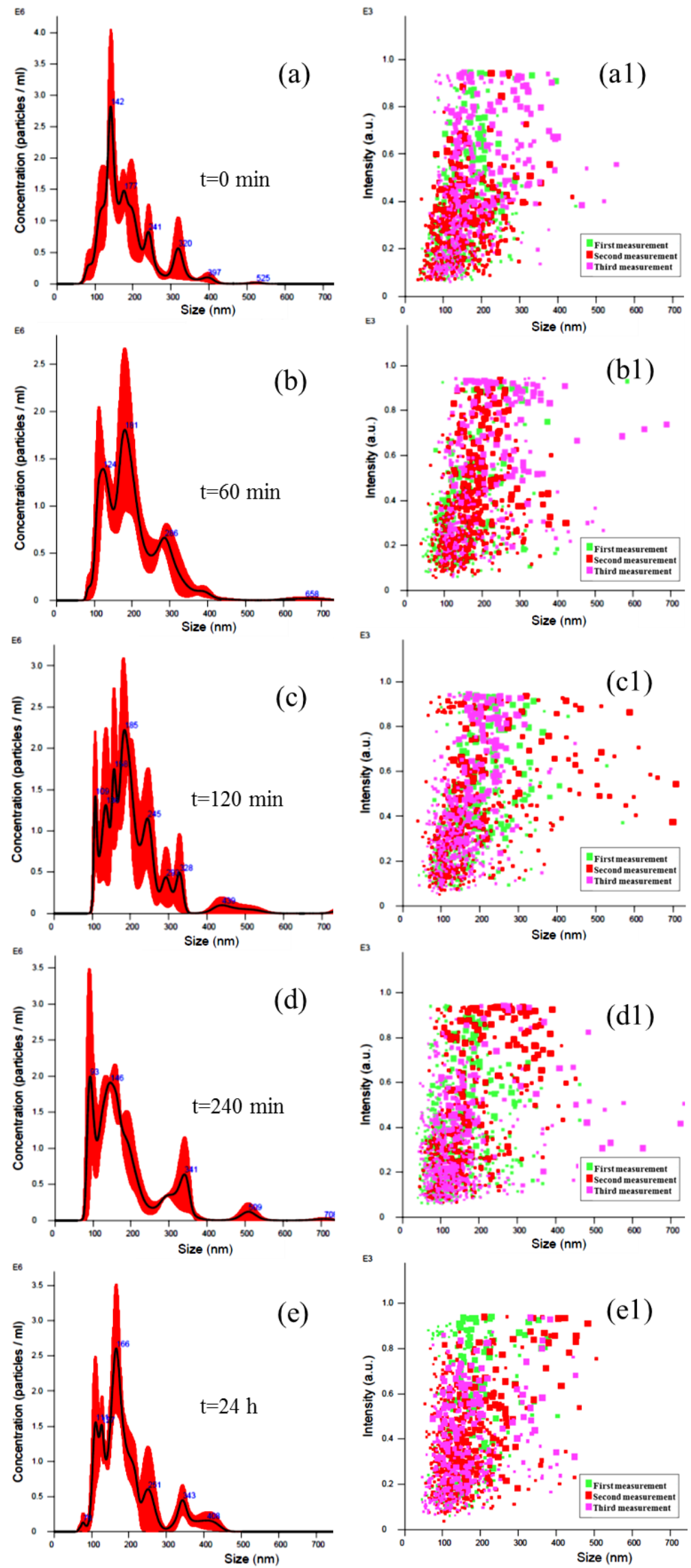

Fig. 2 Stability of oxygen nanobubbles according to time, (a-e) Concentration / Size 
distribution graph; (a1-e1) Intensity / Size graph for nanobubbles and three colors represent three times measurement.

\subsection{Effect of oxygen nanobubbles on the photodegradation of oxytetracycline}

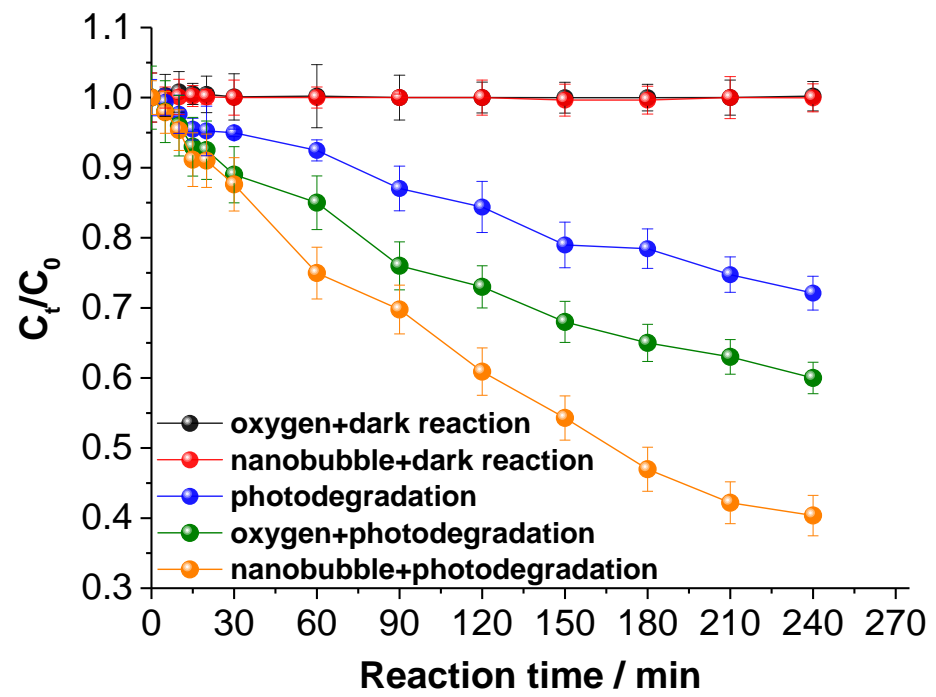

Fig. 3 Effect of oxygen nanobubbles on the photodegradation of OTC

In order to study the synergistic effects of oxygen nanobubbles on the photodegradation of OTC, experiments were carried out under different reaction conditions. As described in the experimental part, all reaction solutions were freshly prepared by using ultrapure water before irradiation. The initial concentration of oxygen nanobubbles and OTC in the reaction solution was $2.0 \times 10^{8}$ particles $/ \mathrm{mL}$ and 20 $\mu \mathrm{M}$, respectively. All experiments were performed at $\mathrm{pH}$ 7.0. Dark reactions were carried out in systems with oxygen nanonbubbles and ordinary oxygen aeration, respectively. The initial concentration of dissolved oxygen (DO) was $30 \mathrm{mg} / \mathrm{L}$ in both systems. The bubbles concentration in the ordinary oxygen aeration system was only $8.8 \times 10^{6}$ particles $/ \mathrm{mL}$ (Fig. S1). The macro or micro-bubbles were easily dissolved into the reaction solution. As depicted in Fig. 3, no OTC degradation was detected in the 
dark reaction systems. It was demonstrated that OTC could not be directly degraded by the oxygen nanobubbles or oxygen. The direct photodegradation of OTC was occurred under irradiation and the photodegradation efficiency was $28 \%$ after $4 \mathrm{~h}$. This result was in agreement with the previous study that described the direct photodegradation of OTC under visible light was one of the removal pathways in water [60]. The effects of oxygen nanobubbles and ordinary oxygen aeration on the photodegradation of OTC were also compared at the same initial concentration of DO $30 \mathrm{mg} / \mathrm{L}$. The results presented in Fig. 3 showed that with ordinary aeration of oxygen, the photodegradation efficiency was about $40 \%$ after $4 \mathrm{~h}$ reaction, but in the presence of the oxygen nanobubbles the photodegradation efficiency increased to $60 \%$. The kinetics analysis showed that the OTC degradation fitted well with the pseudo-first-order correlation and the $k$ value for OTC photodegradation in the presence of oxygen nanobubbles was 1.8 fold higher than that of oxygen aeration (Table 1, Fig. S2). These results showed that introduction of oxygen nanobubbles into the AOP operation for OTC degradation enhanced the photodegradation efficiency. The observed improvement can be ascribed to three factors viz: (a) the role of oxygen in the photodegradation process, which further helped the formation of reactive radicals that caused the degradation of OTC; (b) nanobubble presents higher stability and can provide oxygen into water slowly and last longer time when compare with the ordinary oxygen aeration (Fig. S3), which means that nanobubble can supply more oxygen and with higher efficiency during the photoreaction process; (c) nanobubbles surface effect, which can improve the efficiency of mass transfer during the photodegradation process. It should be noted that during the 
nanobubble collapse, reactive radicals, such as hydroxyl radicals [34, 39], are also generated, which may be effective in the OTC degradation. However, the results presented in Fig. 3 showed that OTC degradation did not occur in the dark condition, thus only nanobubbles could not induced the degradation of OTC without irradiation.

Table 1. Kinetics analysis of the photodegradation of OTC

\begin{tabular}{ccc}
\hline Reaction system & $K\left(\times 10^{-3}, \mathrm{~min}^{-1}\right)$ & Correlation coefficient $R^{2}$ \\
\hline Oxygen + dark reaction & 0.01 & 0.20 \\
Nanobubble + dark reaction & 0.01 & 0.24 \\
Photodegradation & 1.34 & 0.99 \\
Oxygen + photodegradation & 2.14 & 0.98 \\
Nanobubble + photodegradation & 3.92 & 0.99 \\
\hline
\end{tabular}

$k\left(\min ^{-1}\right)$ : reaction rate constant

\subsection{Effect of $p H$}

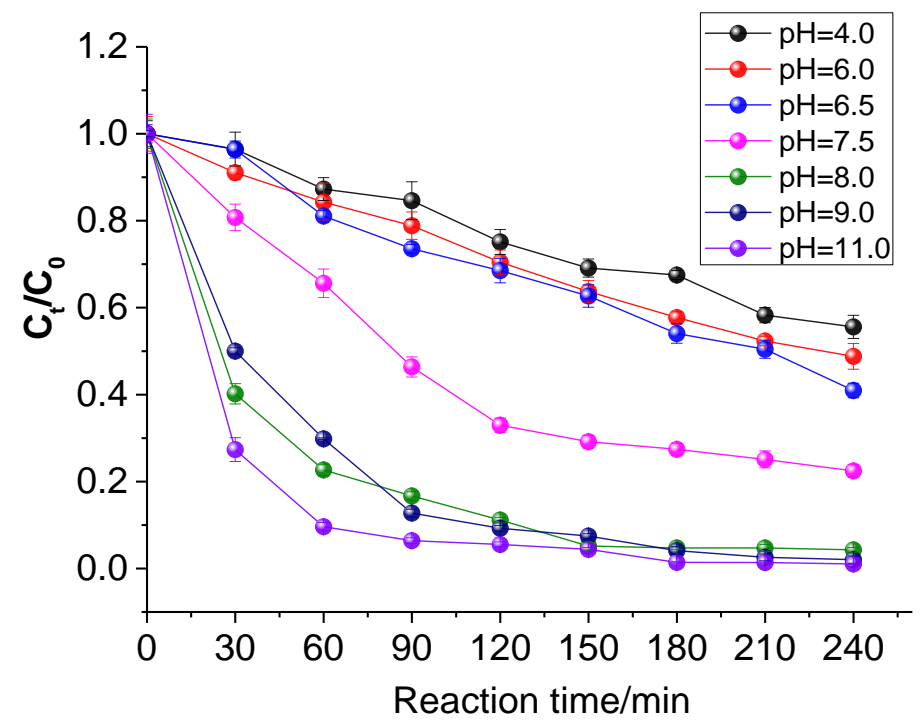

Fig. 4 Effect of $\mathrm{pH}$ on the photodegradation of OTC

In photochemical reactions, the $\mathrm{pH}$ value of the reacting mixture can play an important role. The influence of solution $\mathrm{pH}$ value on the process was determined at constant concentrations of the OTC $(20 \mu \mathrm{M})$ and nanobubbles $\left(2.0 \times 10^{8}\right.$ particles $/ \mathrm{mL}$, 
$\mathrm{pH}$ 7.0) but varied solution $\mathrm{pH}$ values from 4.0 to 11.0 . The photodegradation efficiency of OTC was the initial solution $\mathrm{pH}$ dependent (Fig. 4). The value of the photodegradation efficiency increased from $45 \%$ to $98 \%$, as the solution $\mathrm{pH}$ value increased from 4.0 to 11.0. Consequent upon the affirmed role of nanobubbles in the photodegradation of the OTC (Fig. 3), the stability of the nanobubbles was also monitored at the different operating reaction $\mathrm{pH}$ values. The nanobubbles concentration and size distribution fluctuated with the solution $\mathrm{pH}$ values. The nanobubbles concentration increased from 0.76 to $3.78 \times 10^{8}$ particles $/ \mathrm{mL}$ with increasing $\mathrm{pH}$ value from 3.2 to 11.0 , while the mean size decreased from $205 \mathrm{~nm}$ to $138 \mathrm{~nm}$ as the $\mathrm{pH}$ value increased (3.2-11.0). The higher efficiency of the process at higher $\mathrm{pH}$ value was ascribed to the increase in the concentration of the nanobubbles at higher $\mathrm{pH}$ values and the fact that smaller nanobubbles last a longer time and release more effective oxygen under higher $\mathrm{pH}$ during the reaction process. Zeta potential of oxygen nanobubble was also detected and it was also $\mathrm{pH}$ dependent. It had higher zeta potential (negative) and showed higher stability under higher $\mathrm{pH}$ conditions (Fig. 5c), which was in agreement with the previous report that bubbles with a high absolute value of zeta potential confers stability [11]. 

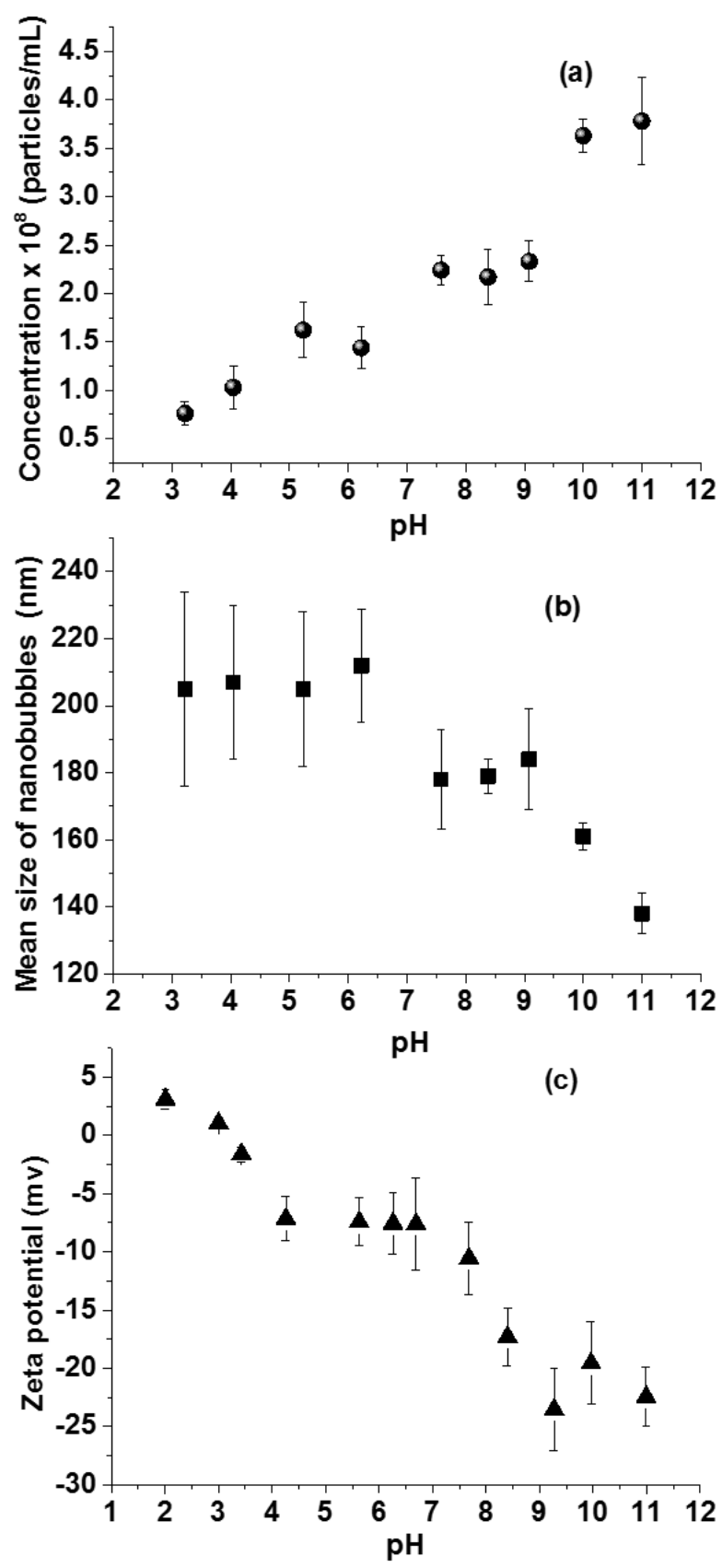

Fig. 5 Variation of concentration (a), size distribution (b), and zeta potential (c) of oxygen nanobubbles according to $\mathrm{pH}$ The aqua phase species of OTC are $\mathrm{pH}$ dependent and the previously reported $\mathrm{pKa}$ values of OTC are 3.57, 7.49 and 9.88 [61]. Thus, it has four species, which include $\mathrm{H}_{3} \mathrm{OTC}^{+} 、 \mathrm{H}_{2} \mathrm{OTC} 、 \mathrm{HOTC}^{-}$and $\mathrm{OTC}^{2-}$, respectively. The OTC exists as protonated and deprotonated forms at $\mathrm{pH} 4.0$ and 7.0, and almost fully deprotonated at $\mathrm{pH} 8.0$ and 11.0. 
As presented in Fig. 6, during the deprotonation process a red shift was found in the UV-vis absorption spectra. Probably, the redshift would have increased the spectrum overlap between the OTC light absorption and visible light, which has favored the direct photodegradation of OTC. Accordingly, the deprotonation of OTC was favorable to the direct photodegradation process [60].

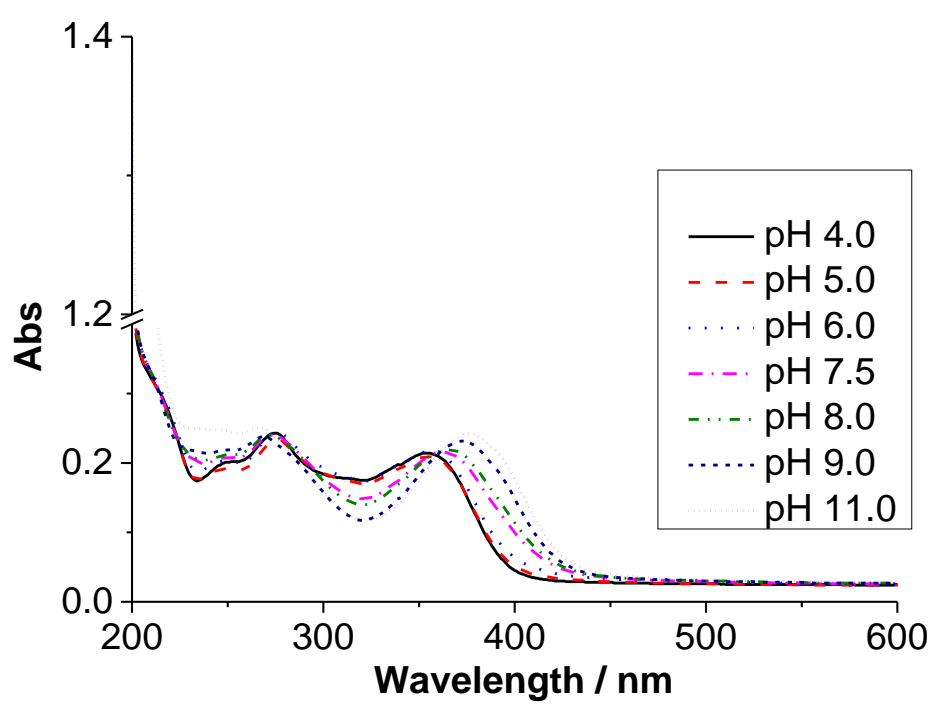

Fig. $6 \mathrm{UV}$-vis absorption spectra of OTC at various $\mathrm{pH} 4.0$ to 11.0

\subsection{Effect of nanobubbles size and concentration}

In order to determine the effects of nanobubbles size and concentration on the reaction, different sizes of nanobubbles were generated by mechanical filtration with teflon membrane with different pore sizes $(0.45 \mu \mathrm{m}$ and $0.22 \mu \mathrm{m})$. The nanobubbles with mean size distributions of $259 \mathrm{~nm}$ (SD $121 \mathrm{~nm}), 202 \mathrm{~nm}$ (SD $74 \mathrm{~nm}$ ) and $173 \mathrm{~nm}$ (SD $39 \mathrm{~nm}$ ) were obtained separately (Fig. 7). Reaction solutions were prepared with the same initial nanobubbles concentration $\left(1.0 \times 10^{8}\right.$ particles $\left./ \mathrm{mL}\right)$ and OTC, but with different bubbles sizes. OTC solution with smaller size nanobubbles showed higher photodegradation efficiency. It can be ascribed to the smaller bubbles have higher 
interfacial area per volume of gas, surface properties and interface effect [32], and these features enhance the surface reaction and improve the efficiency of mass transfer during the photoreaction.
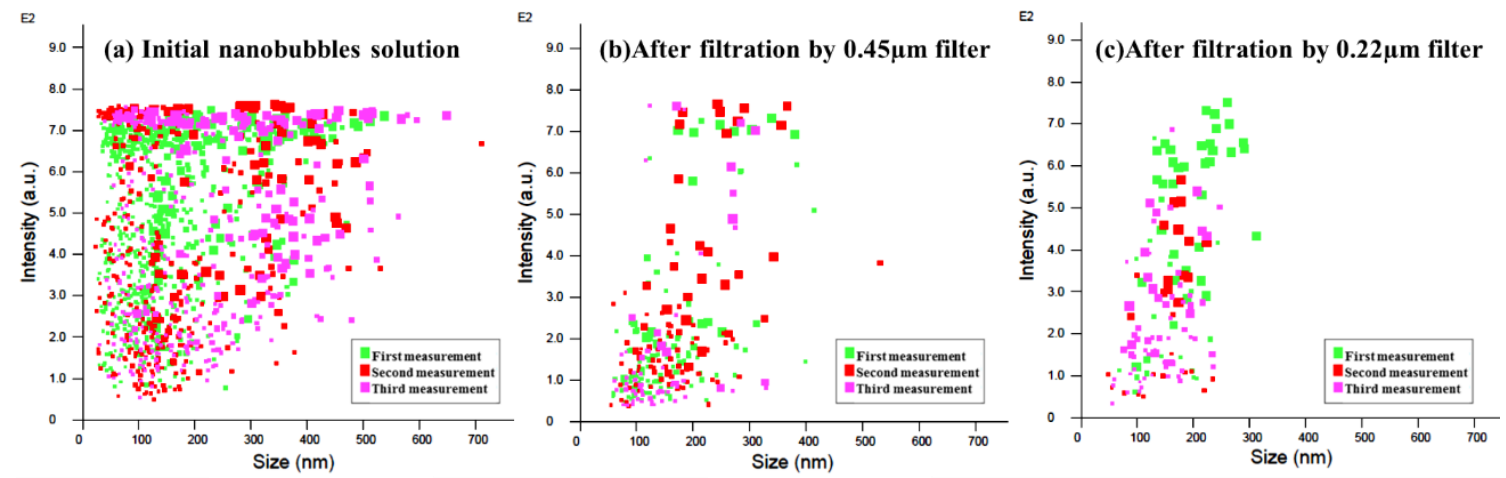

Fig. 7 Intensity / Size distribution graph for nanobubbles and three colors represent

three times measurement.
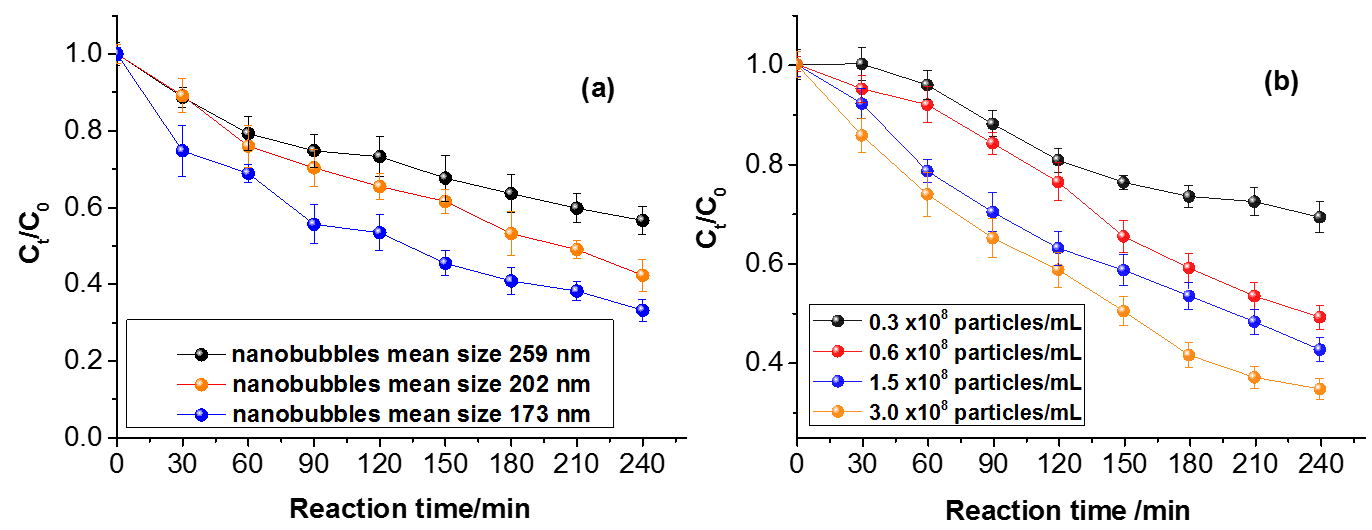

Fig. 8 Effect of nanobubbles size (a) and concentration (b) on the photodegradation of

$$
\text { OTC }(30 \mu \mathrm{M})
$$

The influence of initial nanobubbles concentration that ranged between 0.3 and $3.0 \times 10^{8}$ particles $/ \mathrm{mL}$ was determined at fixed OTC concentration and nanobubbles mean size of $200 \mathrm{~nm}$. The photodegradation efficiency of OTC increased with the increasing nanobubbles concentration (Fig. 8b). The higher concentration of nanobubbles not only support more oxygen during the reaction process but also 
improved the interfacial effect, which strengthens the photodegradation process.

\subsection{Proposed photodegradation mechanisms}

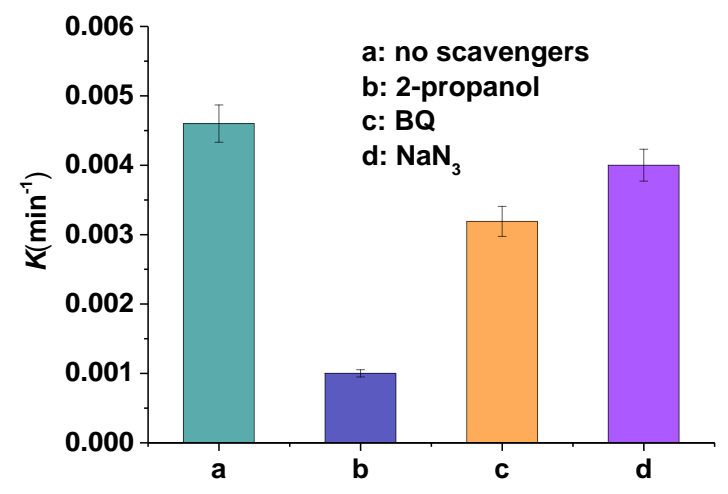

Fig. 9 Active species quenching experiments for degradation of OTC

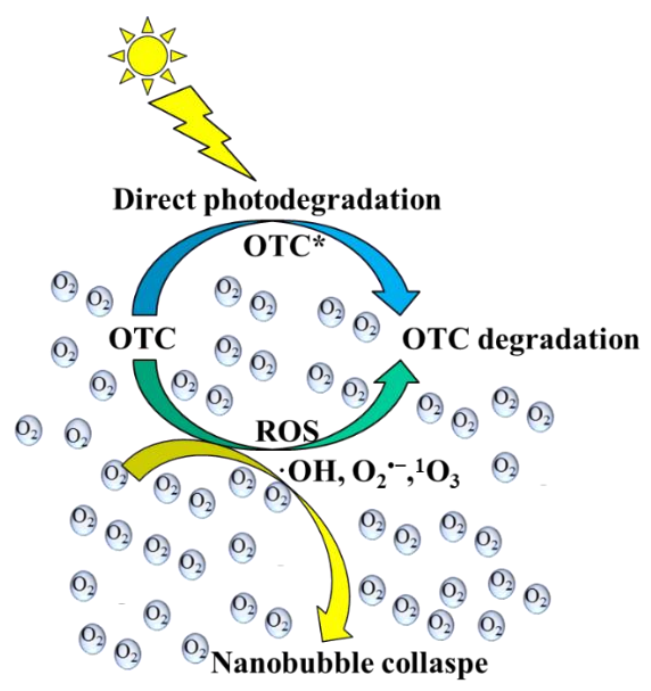

Fig. 10 The proposed mechanism of OTC photodegradation

The quenching experiment was performed to investigate the possible reactive species that induced the photodegradation of OTC. 2-propanol, benzoquinone (BQ) and $\mathrm{NaN}_{3}$ were used as scavengers for trapping hydroxyl radicals $(\bullet \mathrm{OH})$, superoxide radical $\left(\mathrm{O}_{2}{ }^{-}\right)$and singlet oxygen $\left({ }^{1} \mathrm{O}_{2}\right)$, respectively. All experiments were performed at $\mathrm{pH}$ 7.0. The kinetics of OTC photodegradation fit very well with the pseudo-first-order correlation. As shown in Fig. 9, the pseudo-first-order rate constant $(K)$ of OTC 
decreased to $0.001 \mathrm{~min}^{-1}$ when 2-propanol was added, thus indicating that $\bullet \mathrm{OH}$ played the predominant role in the photodegradation reaction. With the addition of BQ, the $K$ slightly increased to $0.003 \mathrm{~min}^{-1}$ compared with that of the 2-propanol group, which confirmed that $\mathrm{O}_{2}{ }^{-}$- was another major active species for OTC degradation. Whereas the presence of ${ }^{1} \mathrm{O}_{2}$ scavenger $\mathrm{NaN}_{3}$ showed little effect, which suggested that ${ }^{1} \mathrm{O}_{2}$ slightly contributed to the inhibition of the photodegradation efficiency. This result was in agreement with the previous reports that $\cdot \mathrm{OH}, \mathrm{O}_{2}{ }^{-}$and ${ }^{1} \mathrm{O}_{2}$ coexisting within nanobubbles solution, while $\cdot \mathrm{OH}$ was a more important oxidant in the system than $\mathrm{O}_{2}$ ${ }^{-}{ }^{-}$or ${ }^{1} \mathrm{O}_{2}$ [35]. It was reported that the long term stability of nanobubbles (> hours) could continuously generate $\bullet \mathrm{OH}[62]$. Since $\bullet \mathrm{OH}$ are nonselective and can oxidize many kinds of organic pollutants, it could be inferred that $\bullet \mathrm{OH}$ is the predominant active species in the photodegradation of OTC.

The proposed mechanism of OTC photodegradation is shown in Fig. 10. Since the OTC species are $\mathrm{pH}$ dependent and the fractions of $\mathrm{HOTC}^{-}$and $\mathrm{OTC}^{2-}$ have visible light absorption (Fig. 6), thus the OTC degradation can be easily triggered through formation of OTC excited state (OTC*) at neutral or alkaline $\mathrm{pH}$ under visible irradiation. During the photoreaction process, oxygen is consumed and the more required oxygen can change the mass balance around oxygen nanobubbles and thus caused the collapse of oxygen nanobubbles to supply more active oxygen. As shown in Fig. S4, the oxygen nanobubble concentration was decreased from $2.0 \times 10^{8}$ to $0.9 \times$ $10^{8}$ particles $/ \mathrm{mL}$ after $4 \mathrm{~h}$ reaction. ROS such as $\bullet \mathrm{OH}, \mathrm{O}_{2}{ }^{-}$and ${ }^{1} \mathrm{O}_{2}$ were generated during the collapse of nanobubbles, which can improve the removal of OTC. The 
synergistic mechanism of oxygen nanobubble/photolysis process is that oxygen nanobubbles supply both dissolved oxygen and ROS to the reaction system, whereas no effect was found in the dark control system without the stimulus of photolysis degradation of OTC.

\section{Conclusions}

For the first time, oxygen nanobubbles were added into the photoreaction system to improve the photodegradation efficiency of OTC under visible light irradiation. From the results, oxygen nanobubbles can be stable exist in the pure water system hours to days, whereas its stability was $\mathrm{pH}$ dependent. Both concentration and size distribution were changed according to $\mathrm{pH}$ from 3.2 to 11.0. The smaller bubbles presented higher photodegradation efficiency, can be attributed to their higher interfacial area per volume of gas and high surface properties. OTC could not be directly degraded by the oxygen nanobubbles without irradiation, while during the photoreaction process, oxygen consumption improved the nanobubble collapse, which can help the generation of ROS. Quenching experiments results demonstrated that $\bullet \mathrm{OH}$ was the predominant active species induced the photodegradation of OTC. Oxygen nanobubbles hold promise to improve the AOPs, such as reduce chemicals usage and costs. This study provides a novel principle and method for the improvement of traditional advanced oxidation technology and nanobubble/AOPs will have an important application in the removal of pollutants with high efficiency.

\section{Acknowledgments}


This work was supported by the National Key R\&D Program of China

(2017YFA0207203), National Natural Science Foundation of China (21976197,

21407160), Science and Technology Service Network Initiative (KFJ-STS-ZDTP-

048), and the Key Research and Development Program of Ningxia (2017BY064).

Conflicts of Interest: The authors declare no conflict of interests.

\section{References}

[1] X.H. Zhang, H. Jun, Nanobubbles at the solid/water interface, Progress in Chemistry 16 (2004) 673681.

[2] V.S.J. Craig, Very small bubbles at surfaces-the nanobubble puzzle, Soft Matter 7 (2011) 40-48.

[3] S.H. Oh, J.-M. Kim, Generation and stability of bulk nanobubbles, Langmuir 33 (2017) 3818-3823.

[4] N. Ishida, T. Inoue, M. Miyahara, K. Higashitani, Nano bubbles on a hydrophobic surface in water observed by tapping-mode atomic force microscopy, Langmuir 16 (2000) 6377-6380.

[5] S.T. Lou, Z.Q. Ouyang, Y. Zhang, X.J. Li, J. Hu, M.Q. Li, F.J. Yang, Nanobubbles on solid surface imaged by atomic force microscopy, Journal of Vacuum Science Technology B 18 (2000) 2573-2575.

[6] H. Ren, S.R. German, M.A. Edwards, Q. Chen, H.S. White, Electrochemical generation of individual $\mathrm{O}_{2}$ nanobubbles via $\mathrm{H}_{2} \mathrm{O}_{2}$ oxidation, Journal of Physical Chemistry Letters 8 (2017) 2450-2454.

[7] S. Yang, P. Tsai, E.S. Kooij, A. Prosperetti, H.J.W. Zandvliet, D. Lohse, Electrolytically generated nanobubbles on highly orientated pyrolytic graphite surfaces, Langmuir 25 (2009) 1466-1474.

[8] W.B. Zimmerman, V. Tesař, H.C.H. Bandulasena, Towards energy efficient nanobubble generation with fluidic oscillation, Current Opinion in Colloid \& Interface Science 16 (2011) 350-356.

[9] L. Wang, X. Miao, G. Pan, Microwave-induced interfacial nanobubbles, Langmuir 32 (2016) 1114711154.

[10] C. Xu, S. Peng, G.G. Qiao, V. Gutowski, D. Lohse, X. Zhang, Nanobubble formation on a warmer substrate, Soft Matter 10 (2014) 7857-7864.

[11] F.Y. Ushikubo, T. Furukawa, R. Nakagawa, M. Enari, Y. Makino, Y. Kawagoe, T. Shiina, S. Oshita, Evidence of the existence and the stability of nano-bubbles in water, Colloids and Surfaces aPhysicochemical and Engineering Aspects 361 (2010) 31-37.

[12] X.H. Zhang, N. Maeda, J. Hu, Thermodynamic stability of interfacial gaseous states, Journal of Physical Chemistry B 112 (2008) 13671-13675.

[13] G. Pan, B. Yang, Effect of surface hydrophobicity on the formation and stability of oxygen nanobubbles, Chemphyschem 13 (2012) 2205-2212.

[14] H. An, B.H. Tan, Q. Zeng, C.D. Ohl, Stability of nanobubbles formed th the interface between cold water and hot highly oriented pyrolytic graphite, Langmuir 32 (2016) 11212-11220.

[15] H. An, G. Liu, R. Atkin, V.S.J. Craig, Surface nanobubbles in nonaqueous media: looking for nanobubbles in DMSO, formamide, propylene carbonate, ethylammonium nitrate, and propylammonium nitrate, Acs Nano 9 (2015) 7596-7607.

[16] D. Lohse, X. Zhang, Pinning and gas oversaturation imply stable single surface nanobubbles, 
Physical Review E 91 (2015) 031003.

[17] D. Lohse, X. Zhang, Surface nanobubbles and nanodroplets, Reviews of Modern Physics 87 (2015) 981-1035.

[18] B. Bhushan, Y. Pan, S. Daniels, AFM characterization of nanobubble formation and slip condition in oxygenated and electrokinetically altered fluids, Journal of Colloid and Interface Science 392 (2013) 105-116.

[19] K. Kikuchi, A. loka, T. Oku, Y. Tanaka, Y. Saihara, Z. Ogumi, Concentration determination of oxygen nanobubbles in electrolyzed water, Journal of Colloid and Interface Science 329 (2009) 306-309.

[20] Z. Wu, X. Zhang, X. Zhang, J. Sun, Y. Dong, J. Hu, In situ AFM observation of BSA adsorption on HOPG with nanobubble, Chinese Science Bulletin 52 (2007) 1913-1919.

[21] G. Pan, G. He, M. Zhang, Q. Zhou, T. Tyliszczak, R. Tai, J. Guo, L. Bi, L. Wang, H. Zhang, Nanobubbles at Hydrophilic Particle-Water Interfaces, Langmuir 32 (2016) 11133-11137.

[22] P. Bhandari, X. Wang, J. Irudayaraj, Oxygen nanobubble tracking by light scattering in single cells and tissues, Acs Nano 11 (2017) 2682-2688.

[23] N. Matsuki, S. Ichiba, T. Ishikawa, O. Nagano, M. Takeda, Y. Ujike, T. Yamaguchi, Blood oxygenation using microbubble suspensions, European Biophysics Journal with Biophysics Letters 41 (2012) 571578.

[24] N. Matsuki, T. Ishikawa, S. Ichiba, N. Shiba, Y. Ujike, T. Yamaguchi, Oxygen supersaturated fluid using fine micro/nanobubbles, International Journal of Nanomedicine 9 (2014) 4495-4505.

[25] A. Sobhy, D. Tao, Nanobubble column flotation of fine coal particles and associated fundamentals, International Journal of Mineral Processing 124 (2013) 109-116.

[26] S. Calgaroto, K.Q. Wilberg, J. Rubio, On the nanobubbles interfacial properties and future applications in flotation, Minerals Engineering 60 (2014) 33-40.

[27] S. Yoshida, M. Kitano, H. Eguchi, Water uptake and growth of cucumber plants (Cucumis sativus L.) under control of dissolved $\mathrm{O}_{2}$ concentration in hydroponics, Acta Horticulturae 440 (1996) 199-204. [28] K. Minamikawa, M. Takahashi, T. Makino, K. Tago, M. Hayatsu, Irrigation with oxygen-nanobubble water can reduce methane emission and arsenic dissolution in a flooded rice paddy, Environmental Research Letters 10 (2015) 084012.

[29] K. Ebina, K. Shi, M. Hirao, J. Hashimoto, Y. Kawato, S. Kaneshiro, T. Morimoto, K. Koizumi, H. Yoshikawa, Oxygen and air nanobubble water solution promote the growth of plants, fishes, and mice, Plos One 8 (2013) e65339.

[30] A. Gurung, O. Dahl, K. Jansson, The fundamental phenomena of nanobubbles and their behavior in wastewater treatment technologies, Geosystem Engineering 19 (2016) 133-142.

[31] M. Brooks, Impossibubbles, New Scientist 215 (2012) 38-41.

[32] A.J. Atkinson, O.G. Apul, O. Schneider, S. Garcia-Segura, P. Westerhoff, Nanobubble technologies offer opportunities to improve water treatment, Accounts of Chemical Research 52 (2019) 1196-1205. [33] E.D. Michailidi, G. Bomis, A. Varoutoglou, E.K. Efthimiadou, A.C. Mitropoulos, E.P. Favvas, Chapter 4 -Fundamentals and applications of nanobubbles, Interface Science and Technology 30 (2019) 69-99. [34] P. Li, M. Takahashi, K. Chiba, Enhanced free-radical generation by shrinking microbubbles using a copper catalyst, Chemosphere 77 (2009) 1157-1160.

[35] S. Liu, S. Oshita, S. Kawabata, Y. Makino, T. Yoshimoto, Identification of ROS produced by nanobubbles and their positive and negative effects on vegetable seed germination, Langmuir 32 (2016) 11295-11302.

[36] K. Tada, M. Maeda, Y. Nishiuchi, J. Nagahara, T. Hata, Z. Zhao, Y. Yoshida, S. Watanabe, M. Ohmori, 
ESR measurement of hydroxyl radicals in micro-nanobubble water, Chemistry Letters 43 (2014) 19071908.

[37] M. Takahashi, K. Chiba, P. Li, Free-radical generation from collapsing microbubbles in the absence of a dynamic stimulus, Journal of Physical Chemistry B 111 (2007) 1343-1347.

[38] A. Agarwal, W.J. Ng, Y. Liu, Principle and applications of microbubble and nanobubble technology for water treatment, Chemosphere 84 (2011) 1175-1180.

[39] P. Li, M. Takahashi, K. Chiba, Degradation of phenol by the collapse of microbubbles, Chemosphere 75 (2009) 1371-1375.

[40] H.I. Masayoshi Takahashi, Toshiyuki Asano, Hideo Horib, Effect of microbubbles on ozonized water for photoresist removal, Journal of Physical Chemistry C 116 (2012) 12578-12583.

[41] S.K.M. Snigdha Khuntia, Pallab Ghosh, Removal of ammonia from water by ozone microbubbles, Industrial \& Engineering Chemistry Research 52 (2013) 318-326.

[42] L. Hu, Z. Xia, Application of ozone micro-nano-bubbles to groundwater remediation, Journal of Hazardous Materials 342 (2018) 446-453.

[43] G. Shen, X.H. Zhang, Y. Ming, L. Zhang, Y. Zhang, J. Hu, Photocatalytic induction of nanobubbles on $\mathrm{TiO}_{2}$ surfaces, Journal of Physical Chemistry C 112 (2008) 4029-4032.

[44] W. Zhang, G. Li, H. Liu, J. Chen, S. Ma, T. An, Micro/nano-bubble assisted synthesis of Au/TiO ${ }_{2} @ C N T s$ composite photocatalyst for photocatalytic degradation of gaseous styrene and its enhanced catalytic mechanism, Environmental Science-Nano 6 (2019) 948-958.

[45] G.Z. Kyzas, G. Bomis, R.I. Kosheleva, E.K. Efthimiadou, E.P. Favvasa, M. Kostoglou, A.C. Mitropoulos, Nanobubbles effect on heavy metal ions adsorption by activated carbon, Chemical Engineering Journal 356 (2019) 91-97.

[46] P. Kulshrestha, R.F. Giese, D.S. Aga, Investigating the molecular interactions of oxytetracycline in clay and organic matter: Insights on factors affecting its mobility in soil, Environmental Science \& Technology 38 (2004) 4097-4105.

[47] H. Wang, M. Zhang, X. He, T. Du, Y. Wang, Y. Li, T. Hao, Facile prepared ball-like TiO2@ @GO composites for oxytetracycline removal under solar and visible lights, Water Research 160 (2019) 197 205.

[48] Q. Zhen, L. Gao, C. Sun, H. Gong, P. Hu, S. Song, R. Li, Honeycomb-like TiO2@@GO nanocomposites for the photodegradation of oxytetracycline, Materials Letters 228 (2018) 318-321.

[49] Q. Chen, S. Wu, Y. Xin, Synthesis of Au-CuS- $-\mathrm{TiO}_{2}$ nanobelts photocatalyst for efficient photocatalytic degradation of antibiotic oxytetracycline, Chemical Engineering Journal 302 (2016) 377387.

[50] J. Xu, Z. Bian, X. Xin, A. Chen, H. Wang, Size dependence of nanosheet $\mathrm{BiVO}_{4}$ with oxygen vacancies and exposed $\{001\}$ facets on the photodegradation of oxytetracycline, Chemical Engineering Journal 337 (2018) 684-696.

[51] S. Ye, X. Zhou, Y. Xu, W. Lai, K. Yan, L. Huang, J. Ling, L. Zheng, Photocatalytic performance of multi-walled carbon nanotube/BiVO4 synthesized by electro-spinning process and its degradation mechanisms on oxytetracycline, Chemical Engineering Journal 373 (2019) 880-890.

[52] W. Zhao, B. Dai, F. Zhu, X. Tu, J. Xu, L. Zhang, S. Li, D.Y.C. Leung, C. Sun, A novel 3D plasmonic p-n heterojunction photocatalyst: $\mathrm{Ag}$ nanoparticles on flower-like $\mathrm{p}-\mathrm{Ag}_{2} \mathrm{~S} / \mathrm{n}-\mathrm{BiVO}_{4}$ and its excellent photocatalytic reduction and oxidation activities, Applied Catalysis B-Environmental 229 (2018) 171180.

[53] H.M. Lwin, W. Zhan, S. Song, F. Jia, J. Zhou, Visible-light photocatalytic degradation pathway of 
tetracycline hydrochloride with cubic structured $\mathrm{ZnO} / \mathrm{SnO}_{2}$ heterojunction nanocatalyst, Chemical Physics Letters 736 (2019) 136806.

[54] N. Shao, J. Wang, D. Wang, P. Corvini, Preparation of three-dimensional Ag $\mathrm{PO}_{4} / \mathrm{TiO}_{2} @ \mathrm{MoS}_{2}$ for enhanced visible-light photocatalytic activity and anti-photocorrosion, Applied Catalysis B-

Environmental 203 (2017) 964-978.

[55] L. Sun, J. Li, X. Li, C. Liu, H. Wang, P. Huo, Y.S. Yan, Molecularly imprinted Ag/Ag $3 V O_{4} / g-C_{3} N_{4}$ Zscheme photocatalysts for enhanced preferential removal of tetracycline, Journal of Colloid and Interface Science 552 (2019) 271-286.

[56] J.R.T. Seddon, D. Lohse, W.A. Ducker, V.S.J. Craig, A deliberation on nanobubbles at surfaces and in bulk, Chemphyschem 13 (2012) 2179-2187.

[57] G. Pyrgiotakis, J. McDevitt, A. Bordini, E. Diaz, R. Molina, C. Watson, G. Deloid, S. Lenard, N. Fix, Y. Mizuyama, T. Yamauchi, J. Brain, P. Demokritou, A chemical free, nanotechnology-based method for airborne bacterial inactivation using engineered water nanostructures, Environmental Science-Nano 1 (2014) 15-26.

[58] R. Etchepare, H. Oliveira, M. Nicknig, A. Azevedo, J. Rubio, Nanobubbles: generation using a multiphase pump, properties and features in flotation, Minerals Engineering 112 (2017) 19-26.

[59] L. Wang, X. Miao, J. Ali, T. Lyu, G. Pan, Quantification of Oxygen Nanobubbles in Particulate Matters and Potential Applications in Remediation of Anaerobic Environment, ACS omega 3 (2018) 10624-10630.

[60] C. Zhao, M. Pelaez, X. Duan, H. Deng, K. O'Shea, D. Fatta-Kassinos, D.D. Dionysiou, Role of pH on photolytic and photocatalytic degradation of antibiotic oxytetracycline in aqueous solution under visible/solar light: Kinetics and mechanism studies, Applied Catalysis B-Environmental 134 (2013) 8392.

[61] R.A. Figueroa, A. Leonard, A.A. Mackay, Modeling tetracycline antibiotic sorption to clays, Environmental Science \& Technology 38 (2004) 476-483.

[62] S. Liu, S. Oshita, Y. Makino, Q. Wang, Y. Kawagoe, T. Uchida, Oxidative capacity of nanobubbles and its effect on seed germination, Acs Sustainable Chemistry \& Engineering 4 (2016) 1347-1353. 